\title{
utilisation d'un clinomètre à très haute résolution pour l'étude du comportement des ouvrages de génie civil
}

\author{
use of very-high-resolution tiltmeter \\ for the study of the response of civil engineering structures
}

\author{
J.P. ASTE*, P.A. BLUM**(1), J.L. BORDES***, B. MEMIER* , B. SALEH**
}

\section{Résumé}

Un clinomètre à très haute résolution $10^{-10} \mathrm{rad}$, conçu initialement pour l'observation des marées terrestres, a été employé pour l'observation à court terme d'ouvrages de génie civil. Il s'agit du massif d'ancrage des câbles d'un téléphérique et d'un mur de soutènement. On a pu mesurer avec une très grande précision le basculement du massif d'ancrage suivant les sollicitations exercées par les câbles lors des allées et venues des cabines. De même malgré des variations dues aux phénomènes thermiques, on a pu montrer la stabilité du mur de soutènement sous les autres sollicitations. II semble que des appareils de très haute précision utilisés sur des périodes de temps courtes ou limitées peuvent donner des informations significatives du comportement des ouvrages.

\section{Abstract}

A very-high-resolution tiltmeter $\left(10^{-10} \mathrm{rad}\right)$, originally designed for the observation of terrestrial tidal waves, has been used for the short-term monitoring of civil engineering structures, the anchor block for the steel ropes of a telpher railway and a retaining wall. The tilting movement of the anchor block under the loads applied by the ropes during travel of the cars was measured with very high precision. Despite variations due to temperature effects, the stability of the retaining wall under other loadings was demonstrated. It would seem that very high precision instruments used over short or limited periods of time can yield significant information on structural behaviour.

\footnotetext{
- B.R.G.M., Atelier risques naturels et aménagements en monta gne, 29, boulevard du 11-Novembre, 69604 Villeurbane Cédex. $\because$ Institut de physique du globe, 4 , place Jussieu, tour $24,2 *$ étage. 75230 Paris Cédex 05

-.. Telemac, 2, rue Auguste-Thomas, 92600 Asnières.
}

(1) Numéro de contribution I.P.G. 79 . 
En 1957, l'un de nous (BLUM 1958) aboutissait, après de nombreux essais, à la réalisation d'un clinomètre de conception originale, destiné à l'étude expérimentale de la marée terrestre; les éléments intervenant dans la sensibilité et la stabilité de ce clinomètre constituent un ensemble monolithique en silice.

En 1982, un contrat avec l'Agence Nationale pour la Valorisation de la Recherche (ANVAR) permet de développer, en coopération avec la société Telemac, les applications de cet instrument à des problèmes de génie civil :

- Études des effets en surface de la fracturation hydraulique (Ph. JULIEN, B. SALEH et al., 1985) ;

- Stabilité d'ouvrages : murs de soutènement, galerie de mines, piliers;

- Réponse d'ouvrages à des sollicitations : piliers de téléphérique, carrière souterraine chargée, barrages.

Ce sont les résultats obtenus sur le massif d'ancrage du nouveau téléphérique de la Saulire à Courchevel et sur un mur de soutènement, près de Lyon, que nous allons développer ici.

\section{DESCRIPTION ET CARACTÉRISTIQUES DE L'INSTRUMENT}

\subsection{Description}

L'instrument (fig. 1) est constitué d'une partie pendulaire de quelques grammes, suspendue par deux fils en silice (Fs), de diamètre environ $10 \mu$, de quelques centimètres de longueur et réalisés de manière à ce qu'ils permettent à la partie pendulaire de tourner autour d'un axe virtuel, quasi vertical ; les fils sont euxmêmes solidaires d'un bâti (Bs) soudé à une pièce tronconique $(\mathrm{Cn})$ elle-même fixée par percussion dans un trou conique réalisé par rodage (à l'aide d'un rodoir diamanté) dans la roche ou le béton constituant l'ouvrage étudié.

La pièce tronconique assurant la liaison de l'instrument et de l'ouvrage peut être fixée sur une surface horizontale (fig. 1) ou verticale. Sur la partie pendulaire, constituée par un assemblage de fines tiges de silice sont collés un miroir concave $(\mathrm{M})$ et une plaque d'argent (Pa).

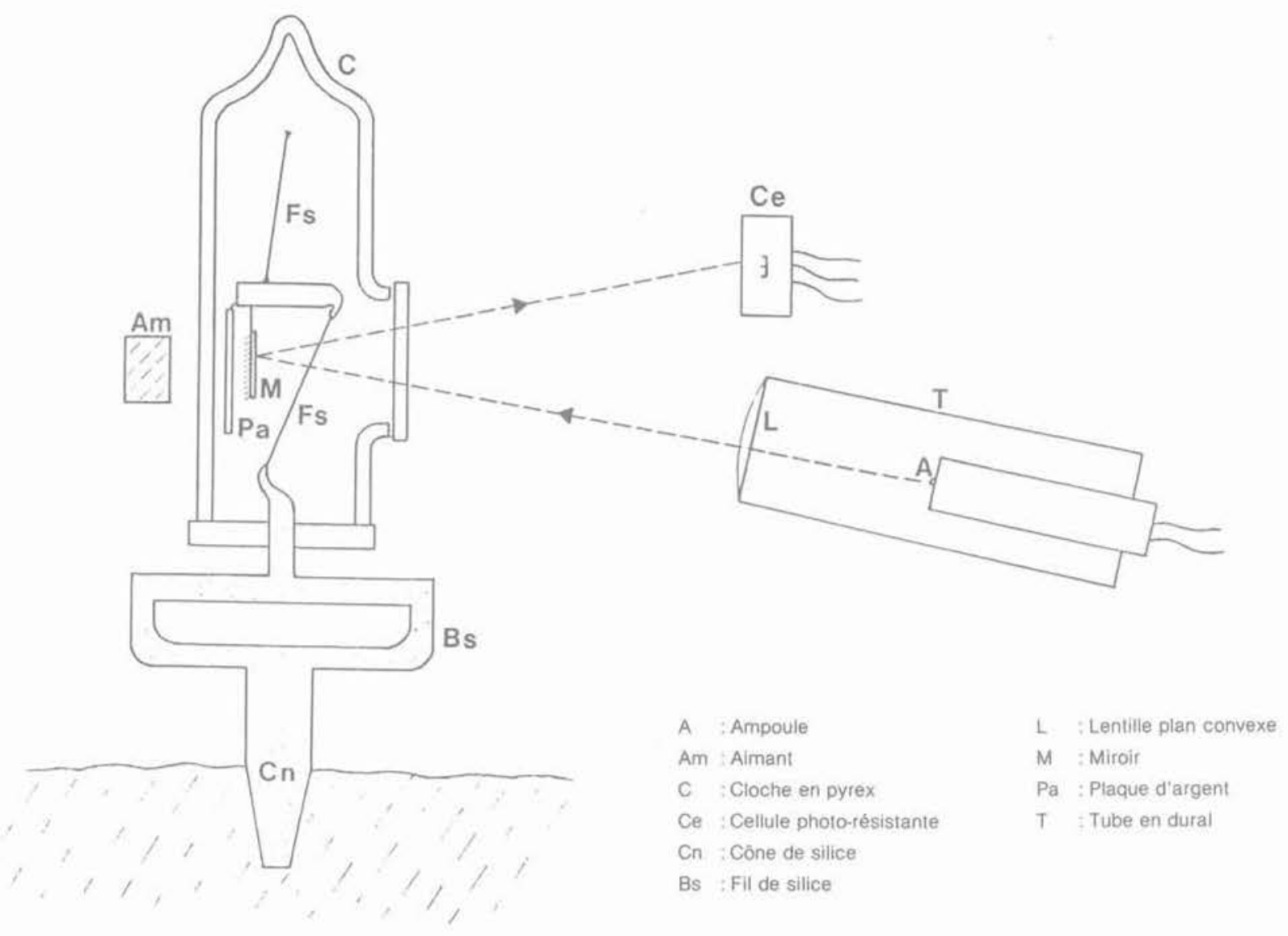

Fig. 1. - Clinomètre en silice. Disposition générale. Coupe. 
Le miroir permet grâce à une lanterne (La) et à une cellule double photorésistante (Cc) (fig. 2) de transformer linéairement la rotation de la partie pendulaire en une variation de tension électrique.

La plaque d'argent, qui constitue l'essentiel de la masse, permet d'amortir le pendule par courant de Foucault grâce à un aimant (Am) placé en son voisinage (fig. 2).

Une rondelle percée et une cloche en pyrex (C) sous vide permettent de protéger le pendule proprement dit et ses fils de suspension.

Pour régler linstrument en sensibilité et en position, on utilise un montage métallique auxiliaire; lorsque l'instrument est approximativement réglé, il est soudé à la pièce tronconique et le montage métallique est enlevé. Le réglage est ensuite affiné par déformation élastique du bâti en silice chargé de masselottes de plomb ou par l'action d'un ressort.

\subsection{Caractéristiques}

Des essais de plusieurs années dans des sites très stables, à température constante, ont montré que la dérive propre de l'instrument est inférieure à $3.10^{-7}$ rd par an, le pouvoir de résolution pouvant atteindre $10^{-10} \mathrm{rd}$.

L'amplification mécanique (c'est-à-dire le rapport entre la rotation de la partie pendulaire et l'inclinaison du sol) peut atteindre 15000 .

Les effets thermiques sur l'instrument lui-même sont toujours largement inférieurs à ceux du matériau sur lequel il est implanté à cause du très faible coefficient de dilatation de la silice.

Dans les applications (de génie civil que nous décrivons ici, l'amplification mécanique peut varier de 200 à 1000 , la résolution maximale pouvant être alors de $5 \cdot 10^{-8} \mathrm{rd}$ à $10^{-8} \mathrm{rd}$ environ. La plage de mesure de l'instrument, sans faire intervenir de réglage est alors de + ou $-1,25 \cdot 10^{-4}$ rd à + ou $-2,5 \cdot 10^{-5}$ rd. Un réglage, automatique ou manuel, effectué â distance, permet d'étendre la plage de mesure. Il est clair que le type d'enregistreur utilisé peut modifier (en les réduisant) la résolution et la plage de mesure. Il faut enfin noter que la sensibilité n'est réglable qu'au moment de l'installation et doit donc être choisie en fonction des inclinaisons escomptées.
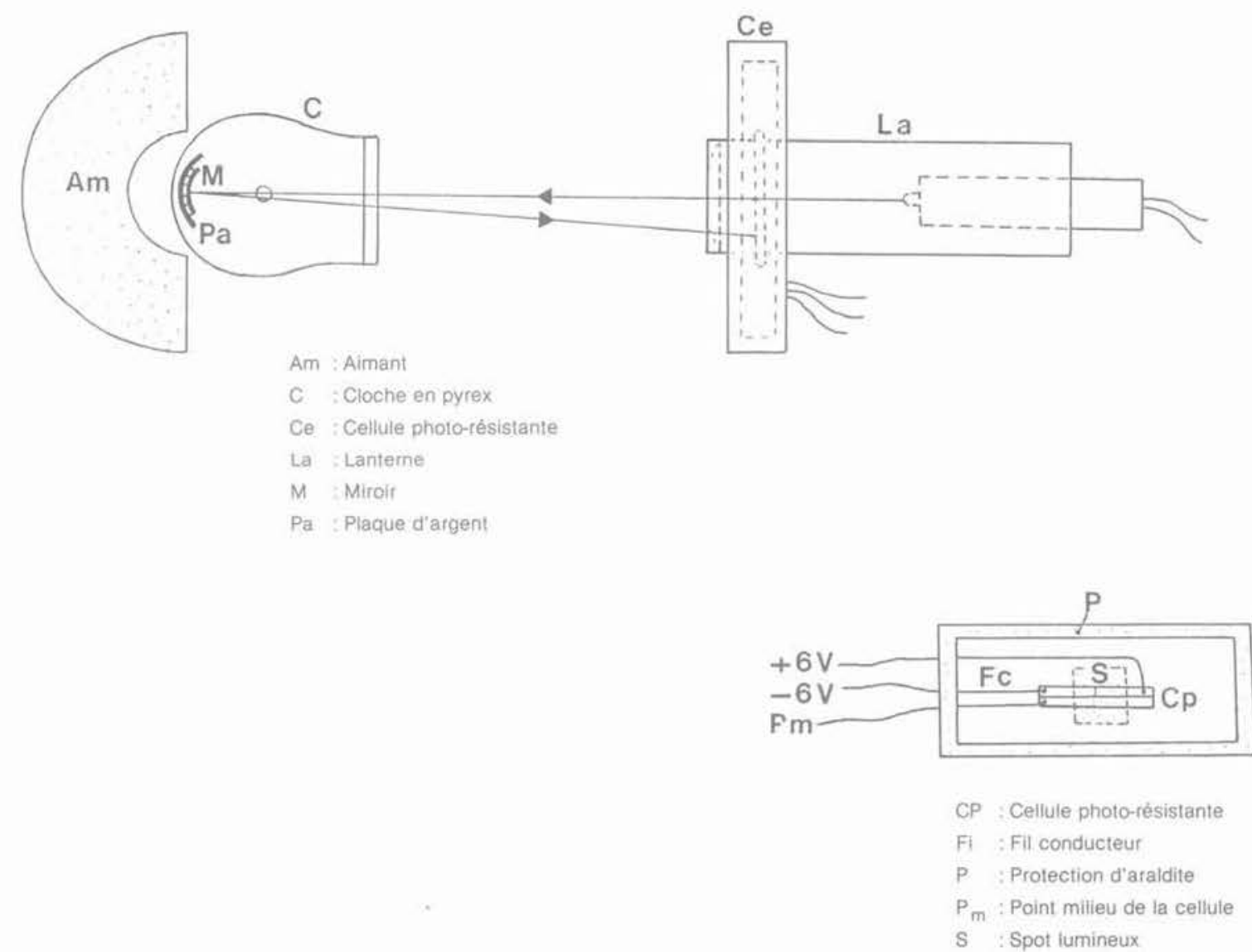

Fig. 2. - Clinomètre en silice. Disposition générale. Vue en plan. 


\section{UN PREMIER EXEMPLE D'APPLICATION : LE TÉLÉPHÉRIQUE DE LA SAULIRE A COURCHEVEL}

\subsection{Description de l'ouvrage}

Il s'agit d'un ouvrage réalisé pour le service public des trois vallées (conseil général de la Savoie) par la cellule des remontées mécaniques de la Direction départementale de l'Équipement de la Savoie en tant que maitre d'œuvre. Le constructeur du matériel est la société Pomagalski. Il s'agit, par certaines de ses caractéristiques du plus grand téléphérique du monde.

Le BRGM est intervenu pour la conception des fondations et dispositifs d'ancrage et l'ensemble de prestations annexes depuis les reconnaissances jusqu'à l'auscultation.

La figure 3 donne les principales caractéristiques de l'ouvrage.

\subsubsection{A la gare inférieure:}

- Sur un terrain de fondation d'origine glaciaire, il fallait reprendre des efforts de $800 \mathrm{t}$ inclinés vers le haut amenés par le téléphérique et des efforts verticaux vers le bas provenant de la superstructure.

L'équilibre général de la structure est assuré par la réaction du sol. La structure comporte une a boîte "à l'arrière remplie de remblais pour compenser les efforts vers le haut. Le radier général mobilisant la réaction du sol est raidi par deux longrines longitudinales et une transversale encastrée dans le sol.

\subsubsection{Au pylône intermédiaire :}

- Le terrain de fondation est d'origine glaciaire enrobant des éboulis. Les efforts sont variables à la fois en direction et en intensité et sollicitent la fondation ou renversement.

L'équilibre est assuré par 4 massifs de béton indépendants enterrés et sur lesquels s'articule la structure métallique.

\subsubsection{A la gare supérieure :}

- Le terrain rencontré est constitué d'un calcaire dolomitique très fracturé par la tectonique et le climat périglaciaire (Trias).

Il fallait reprendre des efforts horizontaux de 1200 t pour l'ancrage et variables en direction et en intensité sur les deux pylônes avant.

L'équilibre est assuré :

- pour les pylônes avant, par deux massifs béton encastrés dans le rocher et cloués par des barres passives de $1.5 \mathrm{~m}$ :

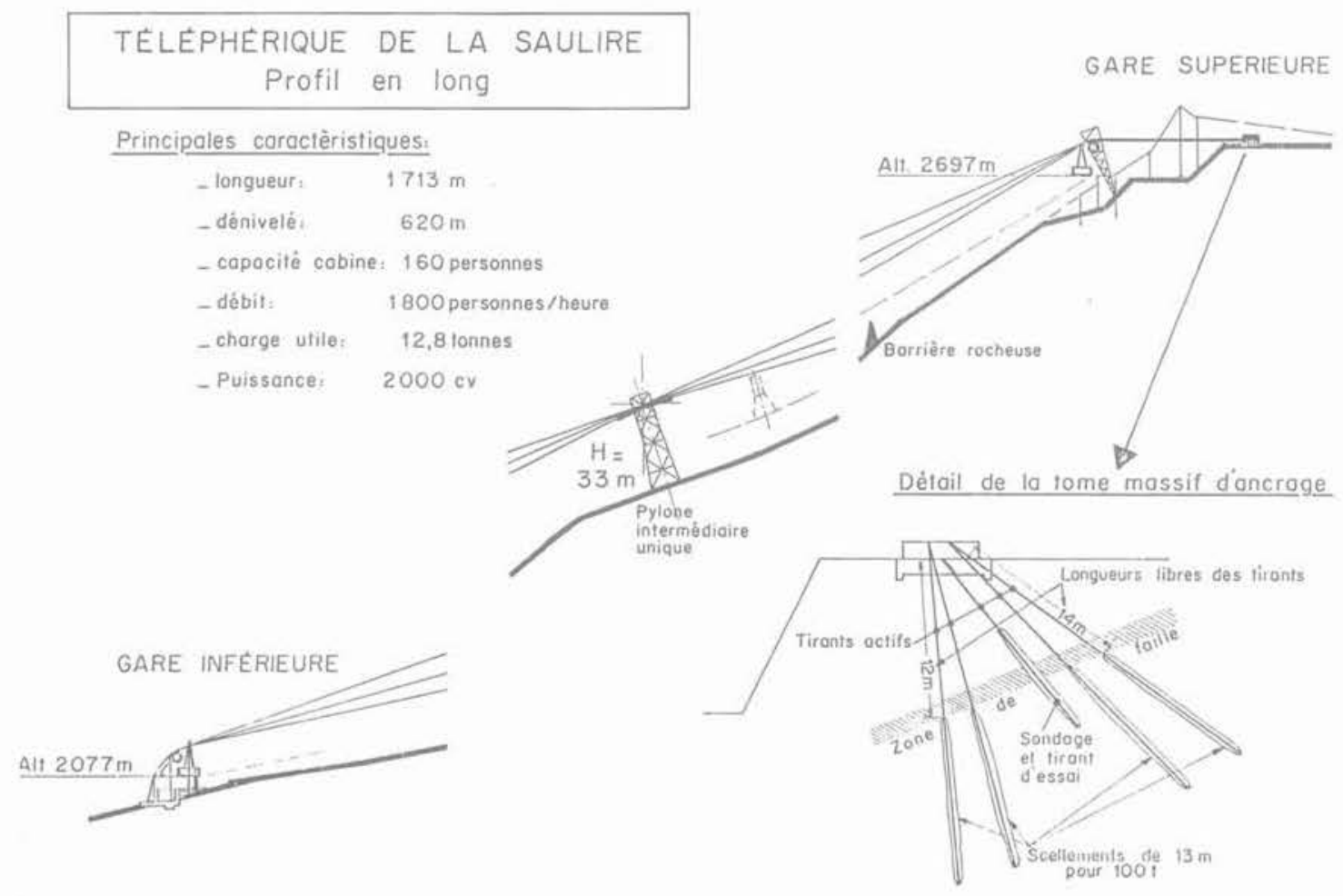

Fig. 3. - Téléphérique de la Saulire. Profil en long. 
- pour la tome d'ancrage, par douze tirants :

- 8 tirants de $120 t$ inclinés à $50 \%$ verticale,

- 4 tirants de $80 t$ inclinés à $20 \%$ verticale.

La longueur d'ancrage est de $13 \mathrm{~m}$, la longueur libre est de $12 \mathrm{~m}$ minimum. Pour augmenter le frottement, la tome d'ancrage est munie de deux longrines ferraillées encastrées de $0,50 \mathrm{~m}$ dans le rocher miné en prédécoupage.

La difficulté consistait principalement à dimensionner un ouvrage stable à la fois en phase intermédiaire (tirants tendus efforts horizontaux normaux) et en phase exceptionnelle (tirants tendus efforts horizontaux, tempête), tout en limitant au maximum les volumes de béton.

Tous les tirants sont munis de cales Glotzl ; on a placé un tube inclinométrique de $30 \mathrm{~m}$; quatre nivelles sur le béton équipent la tome ou massif d'ancrage.

Sur cette tome ont été placés, lors des essais dynamiques de mise en service du téléphérique trois clinomètres type Blum IPG/Paris.

Sur l'ancien téléphérique pour réaliser ces ouvrages, $18000 \mathrm{~m}^{3}$ de terrassements rocheux ont été nécessaires et il a fallu ausculter par distancemétrie, pendant deux ans, la tome d'ancrage (du type poids) de l'ancien téléphérique qui était déchaussée par les terrassements. C'est une solution originale car il n'y a pas de contrepoids.

\subsection{Mesures des inclinaisons}

Sur le massif d'ancrage trois clinomètres ont été implantés, deux longitudinaux (dans la direction de traction des câbles) et un transversal. Les trois instruments sont installés dans une seule cage de protection (cage en PVC, circulaire et isolée par de la laine de verre)

La couverture du massif est assuré par des tôles ondulées non jointives, vent et neige pénétrant à l'intérieur du local.

Les caractéristiques sont données dans le tableau I.

Les essais se divisent en deux parties : un enregistrement permanent pendant les essais du téléphérique, en utilisant un enregistreur potentiométrique SEFRAM, un enregistrement discontinu (1 point toutes les 2 heures), avec une centrale de mesure AOIP.

\subsubsection{Enregistrement permanent pendant les essais}

Les figures 4 et 5 sont particulièrement caractéristiques des résultats obtenus.

Le bruit est inférieur à $10^{-8} \mathrm{rd}$. Les mouvements observés avant déplacement des cabines et d'une période de 5 à 6 secondes sont caractéristiques des micro-séismes.

Après montée et descentes des cabines, on note un parfait retour instantané à la position initiale. Tous les événements survenus au cours du déplacement se tradui-
Courbe inclinométrique enregistrée par l'inclinomètre R8 COURCHEVEL R8C 19/12/84

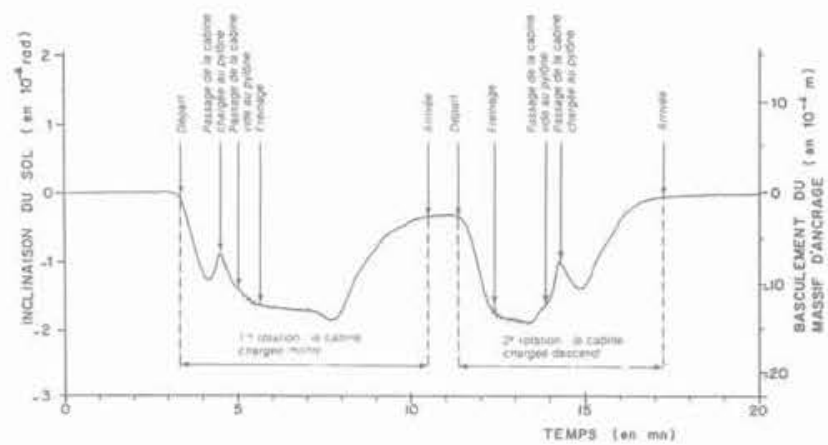

Fig. 4. - Basculement longitudinal, une cabine est chargée.

Courbe inclinométrique enregistrée par l'inclinomètre R8 COURCHEVEL R8NC 19/12/84

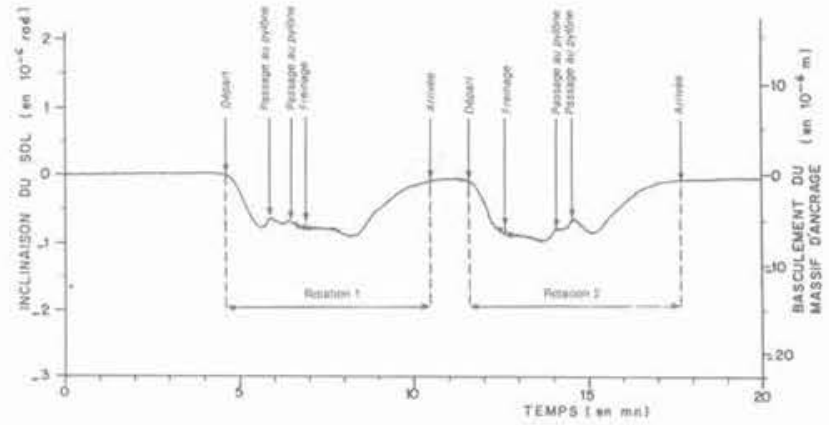

Fig. 5. - Basculement longitudinal, cabines vides.

sent par un signal très clair sur la courbe de réponse de la rotation de la structure d'ancrage.

La comparaison des deux clinomètres en position longitudinale, d'une amplification des oscillations différentes à cause d'un amortissement différents, donnent des courbes superposables (fig. 6).

Courbes inclinométriques enregistrées par les inclinomètres R8 et R5

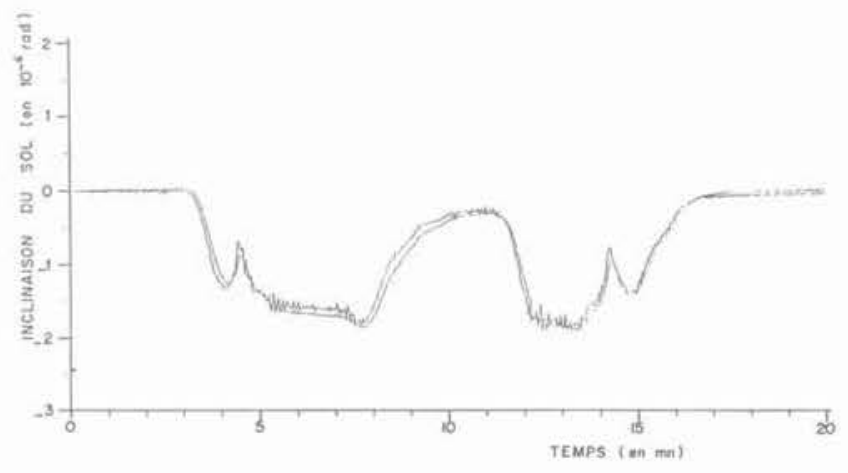

Fig. 6. - Basculement longitudinal, une cabine est chargée. 
TABLEAU 1

\begin{tabular}{|c|c|c|c|c|c|c|c|c|}
\hline \multicolumn{9}{|c|}{ CARACTEERISTIQUES DES CLINOMĖTRES UTILISÉS } \\
\hline CLINOMETRE $N^{\circ}$ & $\begin{array}{c}P E R I O D E \\
(T) \\
(s)\end{array}$ & $\begin{array}{c}\text { CTE } \\
\text { APPAREILS } \\
(K) \\
\left(s^{-2}\right)\end{array}$ & $\begin{array}{c}\text { DIST. } \\
M-C \\
(\mathrm{~L}) \\
(\mathrm{mm})\end{array}$ & $\begin{array}{c}\text { CTE ETAL. } \\
\text { TERRAIN } \\
(C) \\
(\mu r d / v)\end{array}$ & $\begin{array}{c}\text { SENSIBI- } \\
\text { LITE } \\
(S) \\
(v / \mu r d)\end{array}$ & $\begin{array}{c}\text { POUVOIR } \\
\text { DE RESSOL. } \\
(P) \\
\left(10^{-8} r d^{*}\right)\end{array}$ & $\begin{array}{c}\text { PLAGE DE } \\
\text { MESURE } \\
(E) \\
(\mu r d)\end{array}$ & $\begin{array}{l}\text { AMPLIFI- } \\
\text { CATION } \\
\text { MECANI- } \\
\text { QUE }\end{array}$ \\
\hline $\begin{array}{c}R 8 \\
n^{\circ} 509\end{array}$ & 7,2 & 19,2 & 100 & 7,551 & 0,132 & 1,10 & 34 & 1003 \\
\hline $\begin{array}{c}R 5 \\
n^{\circ} 505\end{array}$ & 4,7 & 19,8 & 104 & 9,676 & 0,103 & 2,20 & 43 & 440 \\
\hline $\begin{array}{c}T 5 \\
n^{\circ} 511\end{array}$ & 5,4 & 21,9 & 105 & 8,501 & 0,118 & 1,50 & 38 & 644 \\
\hline
\end{tabular}

Enfin, le basculement transversal du bloc d'ancrage est pratiquement nul (fig. 7). La figure 8 montre un ensemble de cycles sur plusieurs heures.

Courbe inclinométrique enregistrée par linclinomètre T5

COURCHEVEL TSC 19/12/84

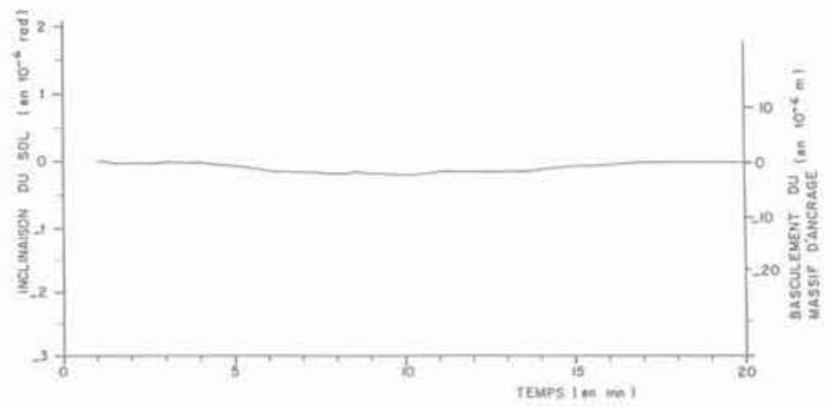

Fig. 7. - Basculement transversal, une cabine est chargée.

Courbe inclinomètrique enregistrẻe par l'inclinomètre R5

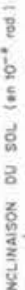
COURCHEVEL R5B 19/12/84

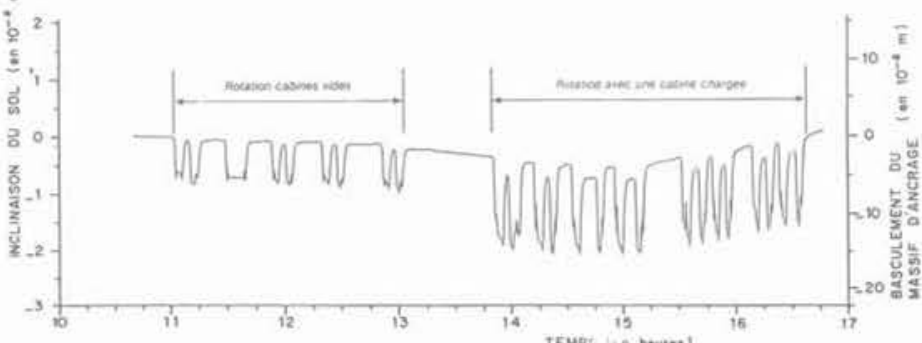

Fig. 8. - Basculement longitudinal, essais dynamiques du 19 décembre 1984.

\subsubsection{Enregistrement sur un mois}

Les figures 9 et 10 montrent la remarquable stabilité de l'ouvrage. En effet, seul l'effet thermique affecte les mesures qui une fois corrigées, montrent que la réponse de l'ouvrage ne varie pas.

\section{UN DEUXIÈME EXEMPLE D'APPLICATION : LA STABILITÉ D'UN MUR DE SOUTĖNEMENT ANCIEN EN SITE URBAIN}

Le quartier de Saint-Clair à Caluire, en banlieue de Lyon occupe un versant glaciaire dominant le Rhône. Dans le cadre de la rénovation de ce vieux quartier, le BRGM avait à surveiller la stabilité de vieux murs en maçonnerie, vestiges de fortifications et hauts de plus d'une dizaine de mètres. Un réseau de surveillance constitué par des extensomètres, des nivelles et des clinomètres a été installé. Il a été composé par des inclinomètres en silice.

Deux clinomètres ont été installés côte à côte sur le premier mur, un pour mesurer l'inclinaison selon une direction perpendiculaire au mur (R1) et l'autre pour mesurer l'inclinaison selon une direction parallèle au mur (T1).

Le montage utilisé est le montage sur une paroi verticale mentionné au § 1.1.

Une plaque métallique en alliage léger est fixée sur le mur au moyen de quatre vis. Un cône en silice est fixé 


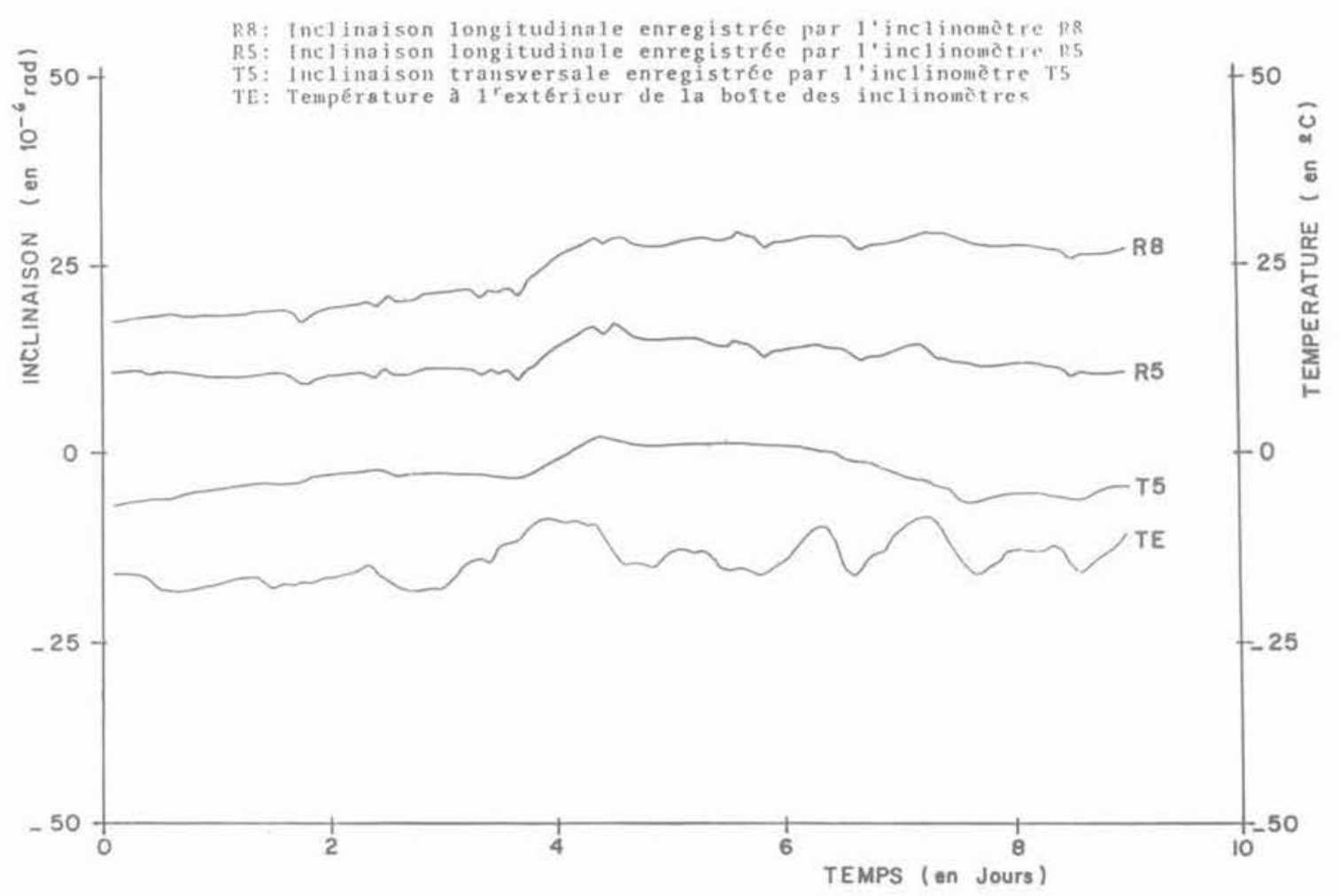

Fig. 9. - Courchevel du 16 au 24 décembre 1984.

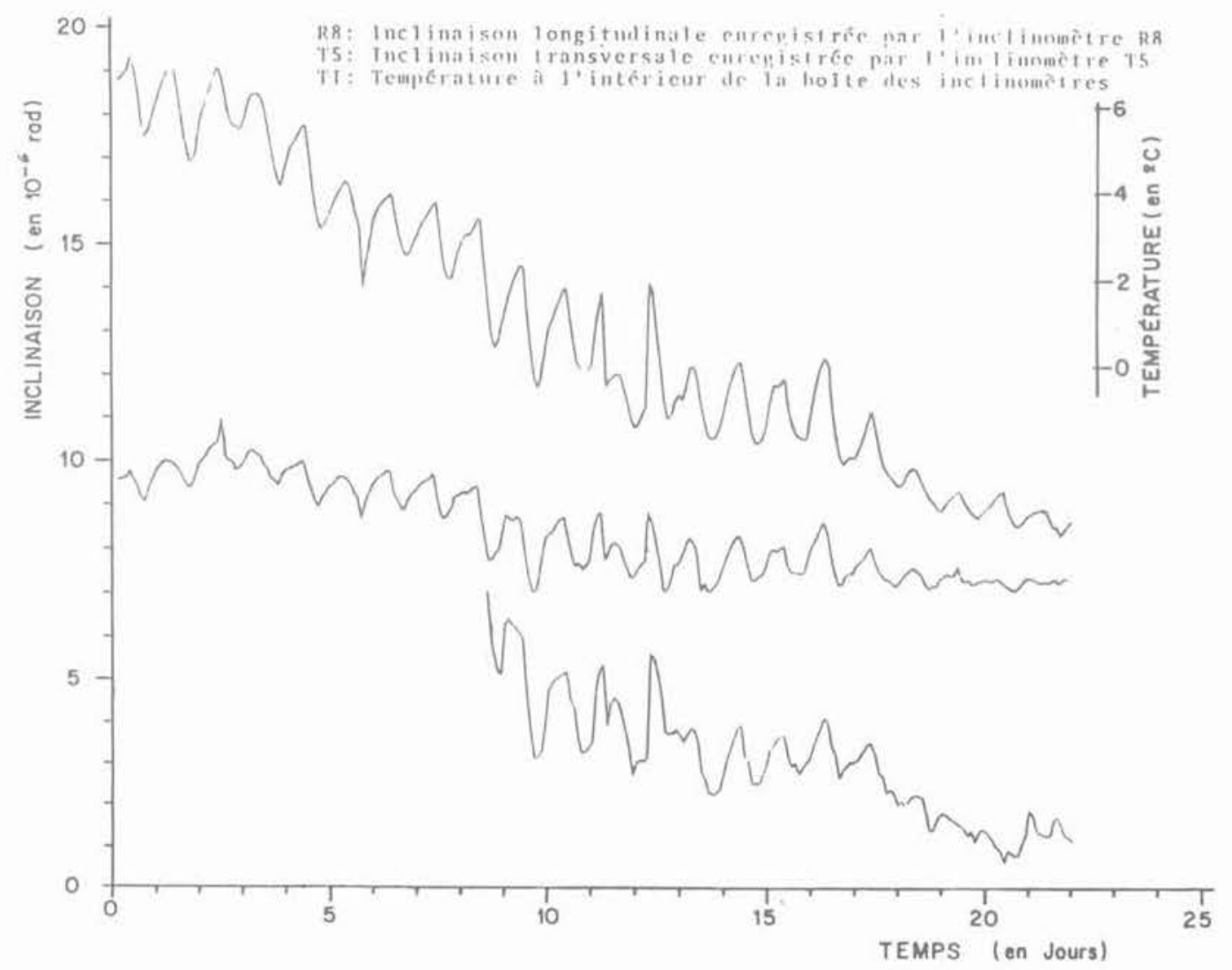

Fig. 10. - Courchevel du 27 janvier au 17 février 1985. 


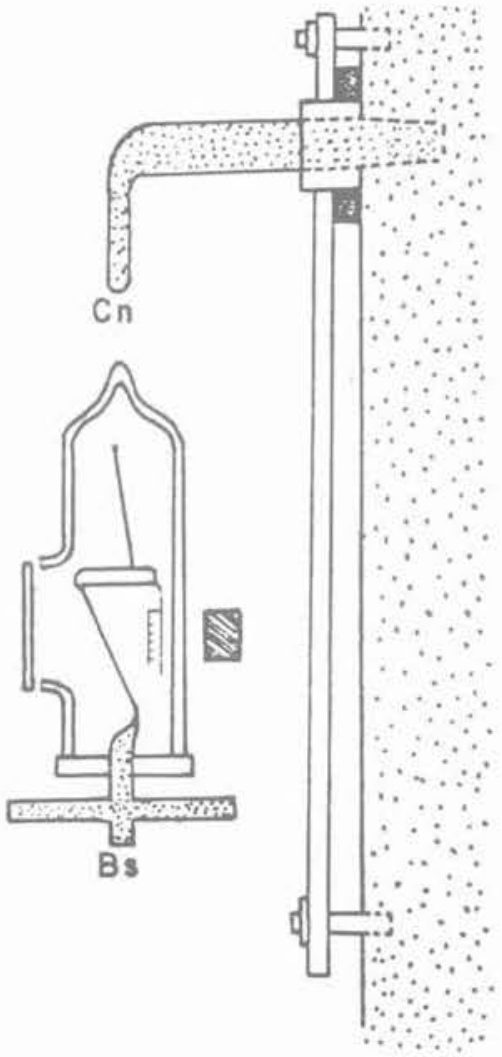

Fig. 11. - Montage du clinomètre sur une face verticale. au mur dans un trou de $10 \mathrm{~mm}$ de diamètre et de $5 \mathrm{~cm}$ de profondeur à travers cette plaque (fig. 11). Le clinomètre est soudé sur le cône. La période de réglage choisie est de l'ordre de 5 secondes. Cette période est relativement faible, car on s'attend à des inclinaisons très grandes. Le maximum d'inclinaison que l'on peut mesurer avant qu'un centrage des spots soit nécessaire est de + ou $-40 \mu \mathrm{rd}$.

L'ensemble des accessoires (cellules, lanterne, électroaimant, ressort) est fixé sur une tige elle-même fixée sur la plaque ${ }^{*}$.

Pour l'enregistrement du signal, un centralisateur de mesure AOIP mis dans une armoire étanche est utilisé pour afficher et imprimer la tension délivrée par chaque clinomètre.

Un deuxième mur a été équipé de façon identique.

Deux sondes de température ont été installées à la première station, une à l'intérieur de la cage, l'autre à l'extérieur.

On dispose de 159 et 184 jours d'enregistrement d'une valeur toutes les deux heures.

La figure 12 donne les données brutes des lectures du clinomètre mesurant les déplacements perpendiculaires au parement du premier mur.

- Cette disposition a été modifiée dans les installations ultérieures. En effet les variations thermiques entraînent des modifications de la pression à l'intérieur de la cage de protection, ce qui déforme la pla. que en entraînant des déplacements de la lanterne et de la cellule. La tige est donc fixée directement dans le mur.

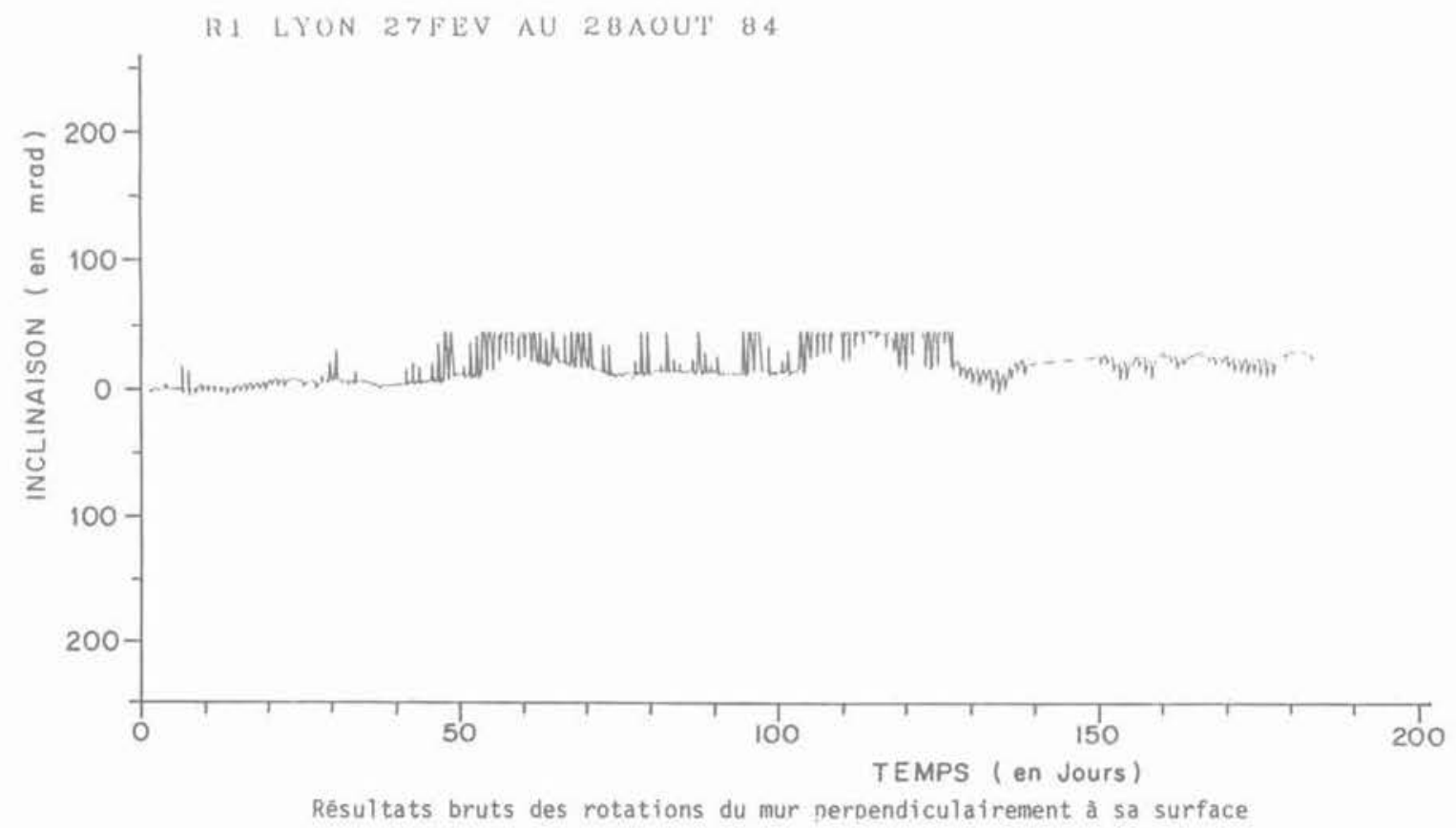

Fig. 12. - Résultats bruts des rotations du mur perpendiculairement à sa surface. 


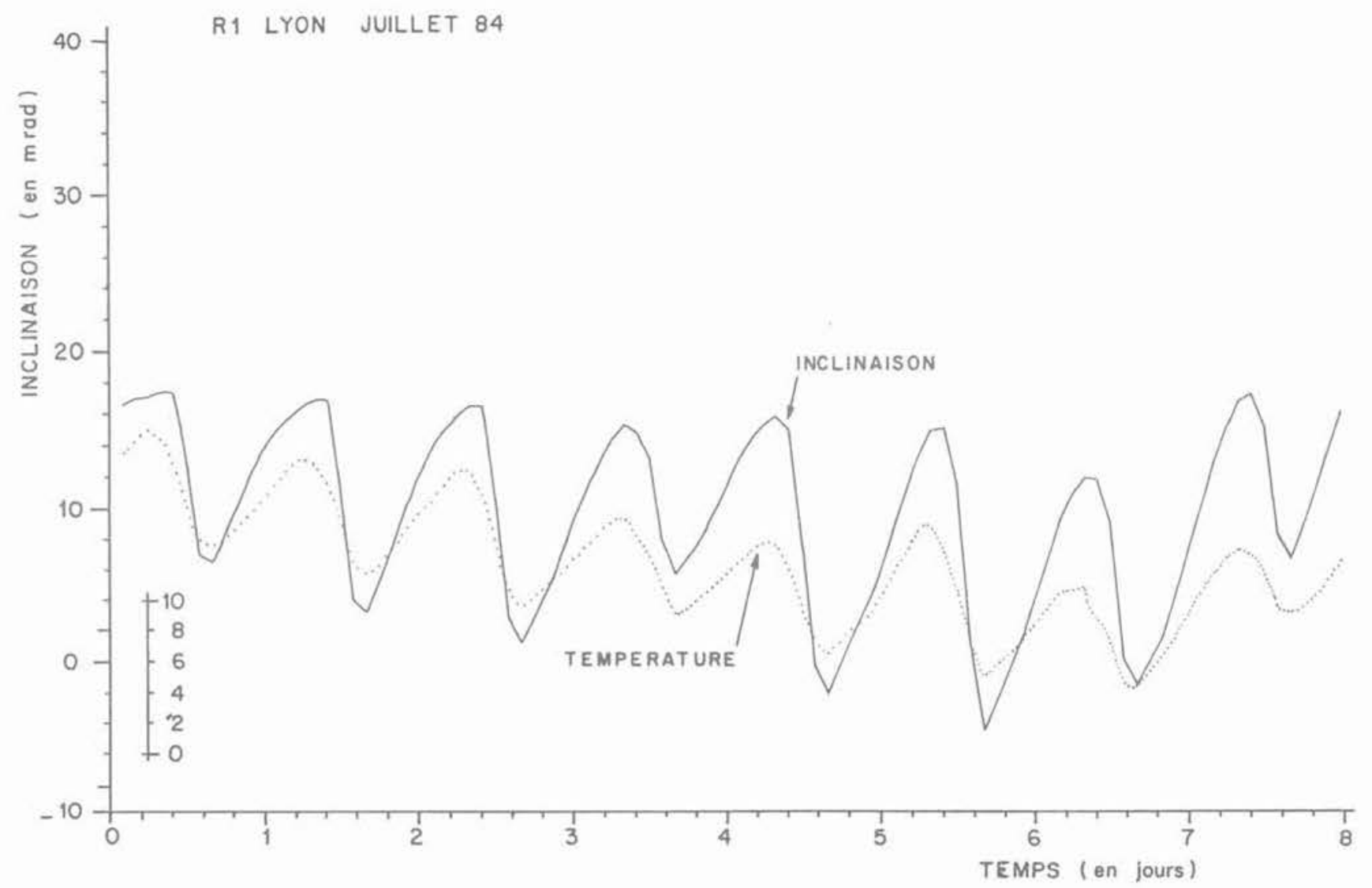

Tempêrature et inclinaison

Fig. 13. - Température et inclinaison.

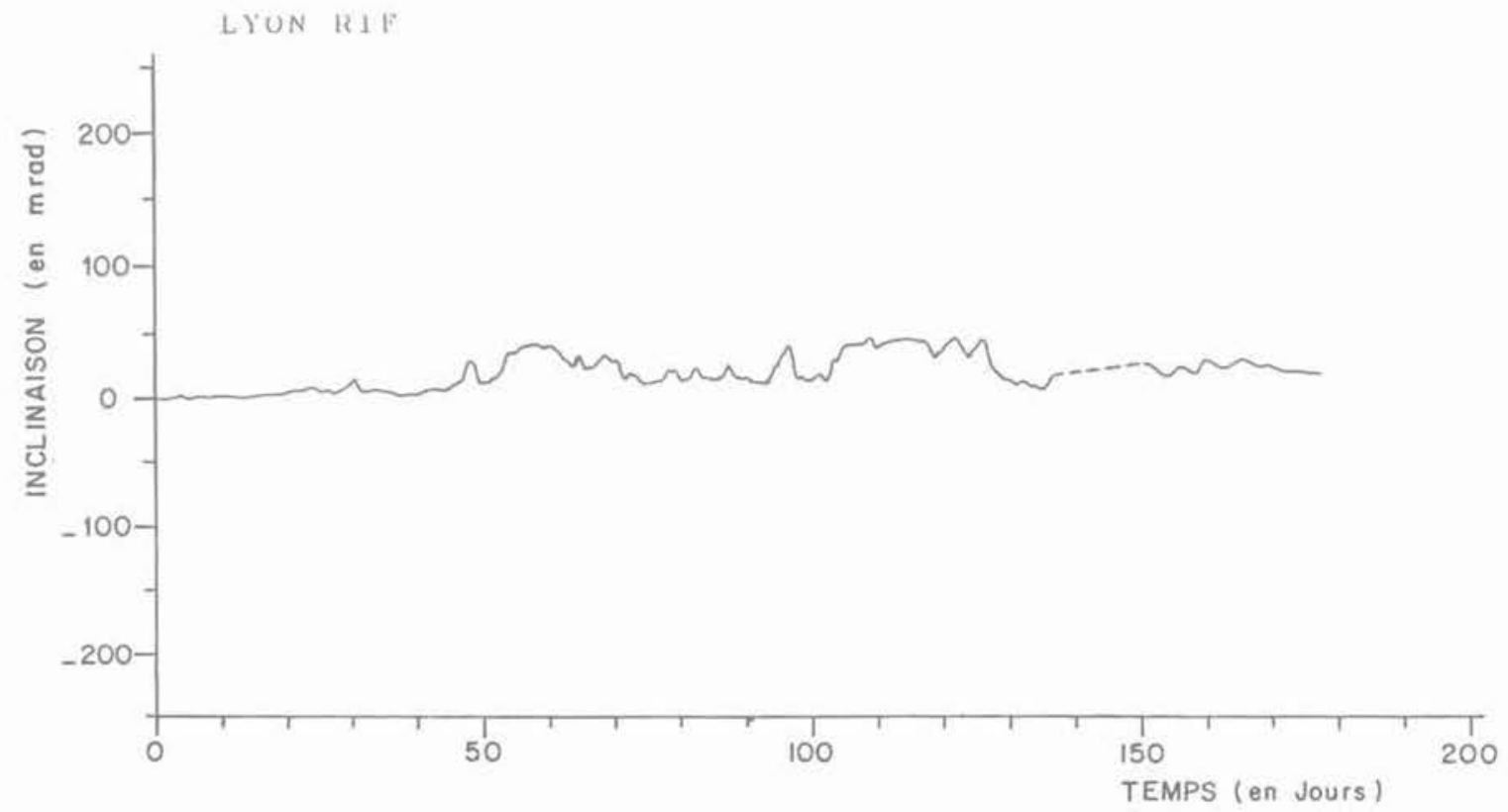

Rotations pernendiculaires au nlan du mur après filtre

Fig. 14. - Rotations perpendiculaires au plan du mur après filtre. 
On remarque sur cette courbe une variation diurne due à la différence de température du mur entre le jour et la nuit. Afin de mettre en évidence ce phénomène on a tracé la courbe d'inclinaison et de température pour huit jours sur la même feuille en inversant la courbe de température en multipliant par un signe négatif (fig. 13). L'examen de ce comportement a permis d'en montrer l'amplitude journalière.

On a pu ensuite étudier les variations à long terme en supprimant les variations diurnes en utilisant un filtre passe bas. La figure 14 donne les valeurs ainsi filtrées. La figure 15 donne les résultats filtrés pour les déplacements parallèles au parement du mur.

On constate que malgré une influence importante de la température, on a pu apporter la preuve de la stabilité des murs, les déplacements au bout de 200 jours étant de l'ordre de quelques micro-radians.

\section{CONCLUSIONS}

L'amplitude des déformations d'un ouvrage de génie civil sous les sollicitations normales qui lui sont appliquées durant sa vie, sont a priori d'un ordre de grandeur bien supérieur à celle des valeurs que peut mesurer un instrument à très haute résolution.

En fait, les exemples que l'on vient de présenter montrent qu'il n'en est pas ainsi et que de tels instruments peuvent apporter des informations très intéressantes.

Il suffit pour cela que l'échelle des temps d'observations soit de quelques heures ou quelques jours, ou que les sollicitations soient faibles, par exemple quand la cabine d'un téléphérique agit sur un massif d'ancrage par l'intermédiaire des câbles porteur et tracteur, ou encore que l'on veuille vérifier la constance de la réponse d'un ouvrage.

D'autre part, la réponse d'un ouvrage à une sollicitation faible mais répétitive permet avec des appareils à très haute résolution d'obtenir la signature d'un ouvrage neuf dont on peut contrôler la non-variation périodiquement pendant la vie de l'ouvrage. Les sollicitations pourraient être soit artificielles par chargement répétitif ou naturelles (vent par exemple). Ce type d'examen pourrait concerner tout ou partie d'un ouvrage.

On peut enfin envisager de contrôler de façon continue avec un système d'alarme les déformations de massif dont l'amplitude de déformation avant rupture est faible. C'est le cas dans les massifs rocheux, et cette approche pourrait particulièrement s'appliquer à un contrôle de la stabilité des falaises.

Toute cette démarche relève d'un emploi à court terme de l'auscultation appliquée aux ouvrages de génie civil. Mais par court terme, on entend non seulement tout ce qui peut relever de la phase des travaux, mais tout ce qui relève d'une courte période d'observations qui permet de porter un diagnostic immédiat sur l'état de l'ouvrage. On peut ainsi y intégrer l'examen de phéno. mène périodique à basse fréquence.

C'est pour obtenir de nouveaux résultats qui permettent d'avancer dans cette voie que des clinomètres ont été mis dans une carrière souterraine creusée dans un massif chargé progressivement en surface par un remblai. D'autres appareils ont été posés dans la galerie de pied d'un barrage-voute pour suivre les réponses de la fondation de l'ouvrage lors du remplissage de la retenue.

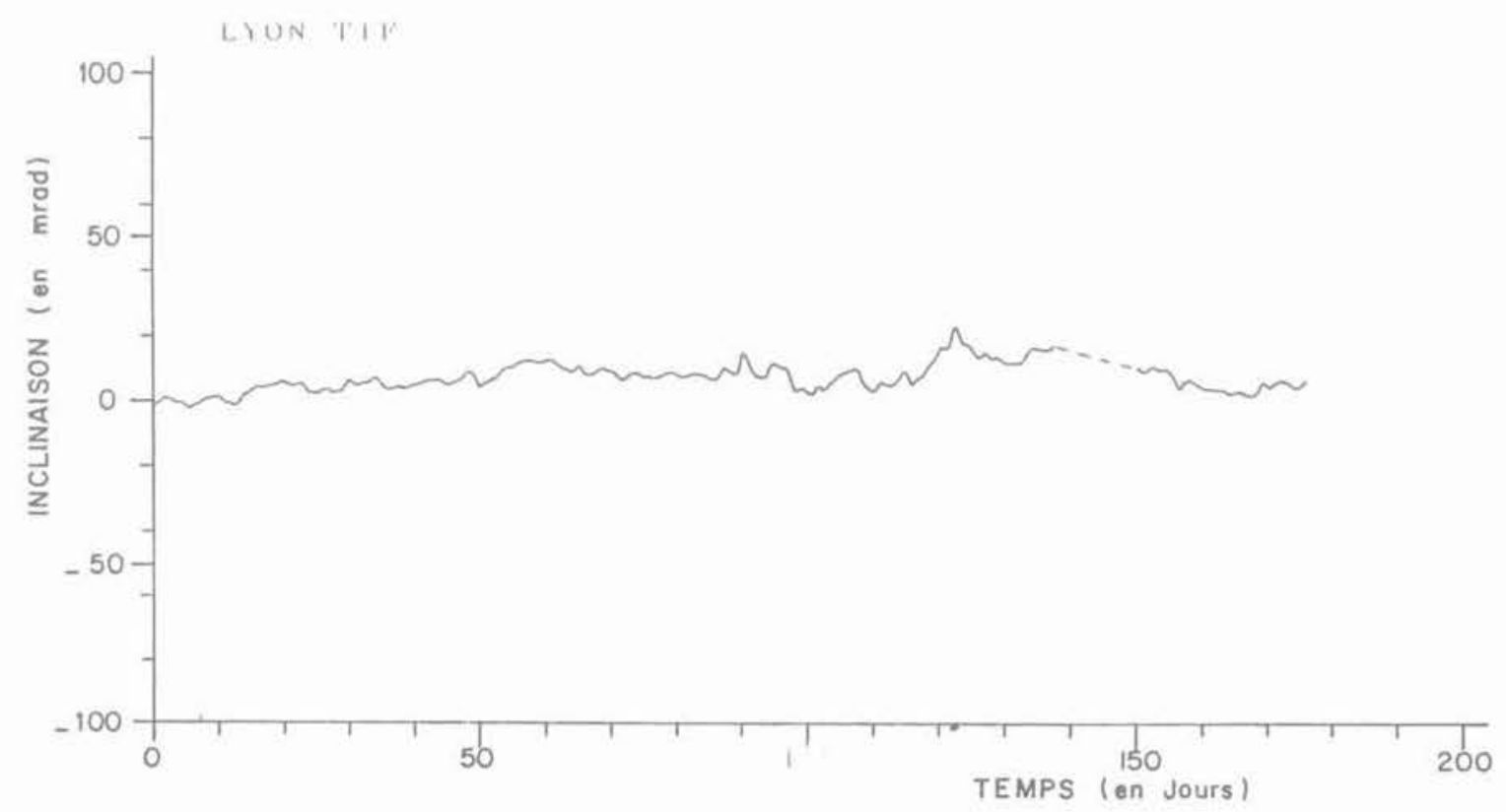

Fig. 15. - Rotation dans le plan du mur après filtre. 
Enfin, des appareils ont été placés dans un laboratoire souterrain.

L'association d'un capteur à très haute résolution et d'une chaîne de saisie et d'acquisition avec le logiciel approprié cornme le permettent les progrès de la micro-informatique doit ouvrir de nouvelles possibilités à l'auscultation dans le domaine du génie civil.

\section{BIBLIOGRAPHIE}

1. P,A. BLUM, Sur un pendule pour la mesure des déviations périodiques de la verticale en un lieu, compte rendu Acamédie des Sciences 246, 2389 , Paris, 1958.

2. Ph. JULIEN, B. SALEH, P.A. BLUM, D., DES$\mathrm{PAX}$, Inversion de données inclinométriques obte. nues au cours de deux expériences de fracturation hydraulique, Revue française de géotechnique, n 31 , Paris, 1985. 
